\title{
Urban Objects Segmentation Using Edge Detection
}

\author{
Sergejs Kodors, Imants Zarembo \\ Rezekne Higher Educational Institution
}

\begin{abstract}
This manuscript describes urban objects segmentation using edge detection methods. The goal of this research was to compare an efficiency of edge detection methods for orthophoto and LiDAR data segmentation. The following edge detection methods were used: Sobel, Prewitt and Laplacian, with and without Gaussian kernel. The results have shown, that LiDAR data is better, because it does not contain shadows, which produce a noise.
\end{abstract}

Keywords - edge detection, remote sensing, segmentation.

\section{INTRODUCTION}

Remote sensing is the science of obtaining and interpreting information from a distance. Remote sensing observes the surfaces and atmosphere of the planets using aerial, ground, satellite and spacecraft observations [7].

The main object of the observations is Earth with purposes: making and updating maps, geospatial databases creation, environmental monitoring, city planning, weather forecasting, etc. [1], [6]-[7].

All remote sensing systems designed to monitor the surface of Earth are based on the reflection and emission of the energy from surface features. Therefore the sensing systems are divided into 3 groups by their information acquisition principles [7]:

- Reflected solar radiation sensors;

- Thermal infrared sensors;

- Imaging radar sensors.

The interpretation of information is connected with image analysis: land use assessment [8], individual trees segmentation [2], etc.

There is a problem that the obtained images contain information about the scene - different objects at once. Therefore there is need to separate these objects before the classification process.

Image segmentation is used to separate the objects and to divide them from a background. Image segmentation is the process of partitioning digital image into multiple regions which have the similar features [3]-[5].

One of the techniques to segment digital images is edge detection.

This manuscript includes information about orthophoto and LiDAR data segmentation using edge detection methods. The Sobel, Prewitt and Laplacian kernels with Gaussian filter were used in the experiment to segment digital image with urban objects.

\section{EdGe DeteCtion PROCESS}

Edge detection process consists of 3 steps [3], [5]:

1) Filtering: this stage goal is to reduce an image noise. The often types of the noise are the salt and pepper noise, the impulse noise and Gaussian noise;

2) Enhancement: this stage goal is to detect changes of intensity in a neighbourhood of a point (pixel). The gradient magnitude are computed to indicate the changes of intensity;

3) Detection: this stage goal is to group all elements into 2 sets: black and white pixels. It is attached by thresholding.

\section{ENHANCEMENT}

The boundaries between different surfaces of the scene in digital image are called edges. The edge is a significant change of image intensity.

One of approaches to estimate the edges is gradientbased methods, where the gradient of 2D function is calculated by the formula (1).

$$
\nabla f(x, y)=\frac{\partial f(x, y)}{\partial x} \bar{i}+\frac{\partial f(x, y)}{\partial y} \bar{k},(1)
$$

where $\bar{i}$ and $\bar{k}$ are unit vectors.

So a point of the significant gradient magnitude (2) is defined as the edge, if the significance is determined by the predefined threshold.

$$
|\nabla f|=\sqrt{(\partial f / \partial x)^{2}+(\partial f / \partial y)^{2}}
$$

where $|\nabla f|$ - the gradient magnitude.

The computation of the partial derivation in the gradient may be approximated in digital image by using masks (see Fig. 1-2) [4].

\begin{tabular}{|l|l|l|}
\hline-1 & 0 & 1 \\
\hline-2 & 0 & 2 \\
\hline-1 & 0 & 1 \\
\hline
\end{tabular}

\begin{tabular}{|c|c|c|}
\hline 1 & 2 & 1 \\
\hline 0 & 0 & 0 \\
\hline-1 & -2 & -1 \\
\hline
\end{tabular}

Fig. 1. Sobel masks. 


\begin{tabular}{|c|c|c|}
\hline-1 & 0 & 1 \\
\hline-1 & 0 & 1 \\
\hline-1 & 0 & 1 \\
\hline
\end{tabular}

\begin{tabular}{|c|c|c|}
\hline 1 & 1 & 1 \\
\hline 0 & 0 & 0 \\
\hline-1 & -1 & -1 \\
\hline
\end{tabular}

Fig. 2. Prewitt masks.

The computation using masks is founded on the convolution (3).

$$
y(n, m)=\sum_{k=-\infty}^{\infty} \sum_{t=-\infty}^{\infty} x(k, t) * h(n-k, m-t),
$$

where $h$ is kernel or mask with the central element $h(0,0)$.

The results of convolution using Sobel or Prewitt masks must be summed by formula (4) or (5) to get the gradient magnitude.

$$
\begin{gathered}
|\nabla f|=\sqrt{G_{x}^{2}+G_{y}^{2}} . \\
|\nabla f|=\left|G_{x}\right|+\left|G_{y}\right| .
\end{gathered}
$$

The Laplacian mask (see Fig. 3) is digital implementation of the second order derivative (6).

$$
\nabla^{2} f=\frac{\partial^{2} f}{\partial x^{2}}+\frac{\partial^{2} f}{\partial y^{2}}
$$

And Laplacian mask 5x5 in Fig. 4.

\begin{tabular}{|c|c|c|}
\hline 0 & -1 & 0 \\
\hline-1 & 4 & -1 \\
\hline 0 & -1 & 0 \\
\hline
\end{tabular}

Fig. 3. Laplacian mask.

\begin{tabular}{|c|c|c|c|c|}
\hline 0 & 0 & -1 & 0 & 0 \\
\hline 0 & -1 & -2 & -1 & 0 \\
\hline-1 & -2 & 17 & -2 & -1 \\
\hline 0 & -1 & -2 & -1 & 0 \\
\hline 0 & 0 & -1 & 0 & 0 \\
\hline
\end{tabular}

Fig. 4. Laplacian mask $5 \times 5$

\section{FILTERING}

The Gaussian filter is one of the methods to reduce the noise.

Gaussian kernel is calculated by the formula (7).

$$
G(x, y)=\frac{1}{2 \pi \sigma^{2}} \exp \left(-\frac{x^{2}+y^{2}}{2 \pi \sigma^{2}}\right)
$$

where $\sigma$ - standard deviation and $G(0,0)$ is the central point of the mask (kernel). Then the image is convolved by Gaussian kernel using the formula (3).
The illustration of 2D Gaussian function is shown in Fig. 5 and Gaussian function shape dependence on the standard deviation in Fig. 6.

\section{POINT Cloud TRANSFORMATION INTO 2D}

LiDAR data is $3 \mathrm{D}$ point cloud, therefore to process it by edge detection, it must be transformed into $2 \mathrm{D}$ image.

Every point of the point cloud has its 3D coordinates XYZ, so it can be transformed into 2D by the point height (see in Fig. 7).

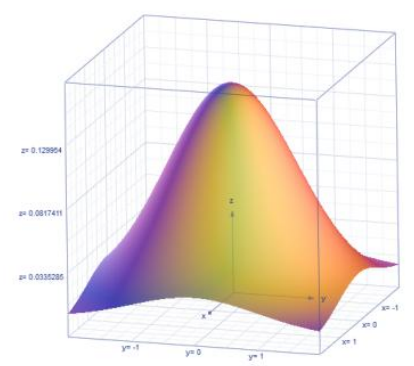

Fig. 5. Gaussian function, if $\sigma=1$.

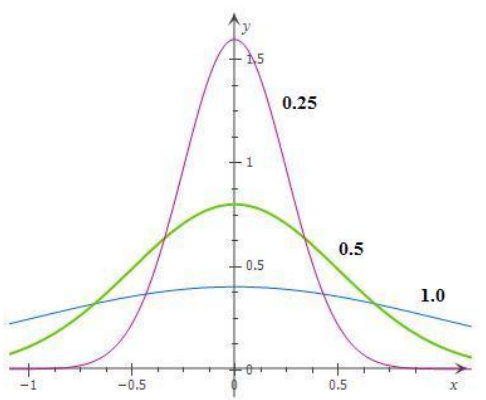

Fig. 6. Shapes of Gaussian function, where bold numbers are standard deviations.

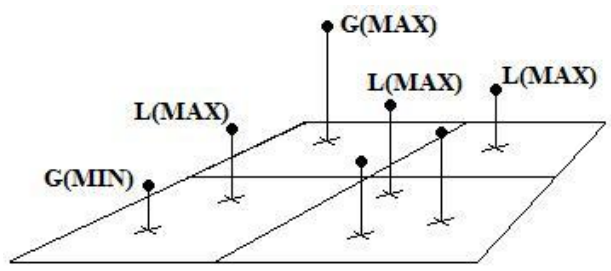

Fig. 7. Point cloud transformation into $2 \mathrm{D}$ by point height, where $G$ are global min \& max points and $L$ are local min \& max points.

Firstly the surface XY is divided into sectors, then the local maximum is found to every sector. After that the sectors are colored in gray colors, where white and black colors are specified by the global minimum and maximum. 


\section{MATERIALS AND METHODS}

The process can be generalized by the following sequence:

1) Image is converted into grayscale format;

2) Gaussian filter $(15 \times 15,3.0)$ is applied;

3) Sobel, Prewitt, Laplacian $3 \times 3$ and $5 \times 5$ filters are applied;

4) Min-max normalization is applied;

5) Thresholding by mean value is applied.

The special patterns have been prepared for the experiment, which simulate orthophoto and transformed LiDAR data into 2D. These patterns are illustrated in Fig. 8.
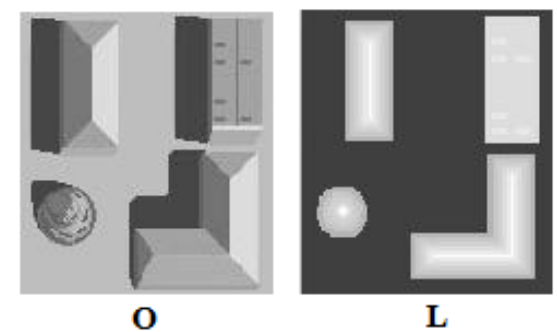

Fig. 8. Patterns for experiment, where $O$ - simulates orthophoto, but $L-$ simulates transformed LiDAR data.

\section{RESULTS AND DISCUSSION}

The results of edge detection are illustrated in Fig. 9-16, where the alphabetic symbols are the first symbols of the kernels names.

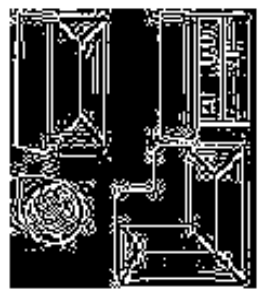

L3x 3

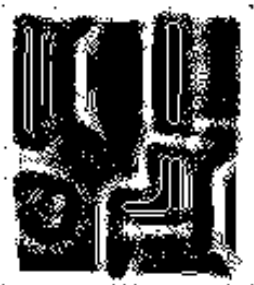

GL3x3
Fig. 9. Laplacian 3x3, pseudo-orthophoto case.

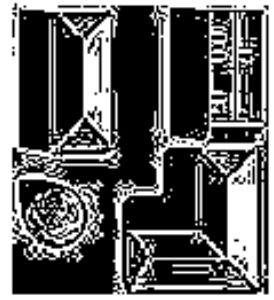

L5x5

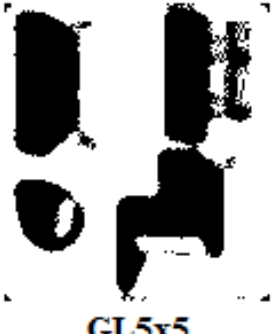

GL5x5
Fig. 10. Laplacian 5x5, pseudo-orthophoto case.

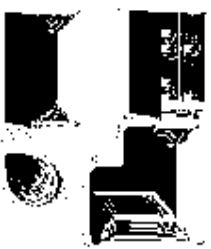

$\mathbf{S}$

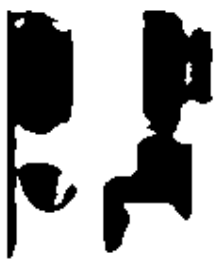

GS
Fig. 11. Sobel, pseudo-orthophoto case
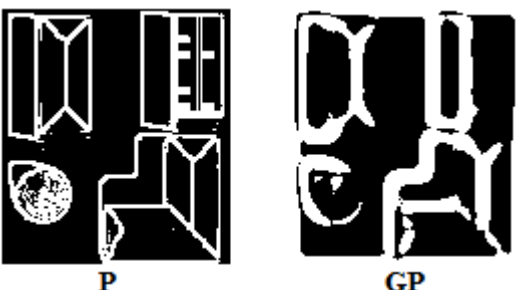

Fig. 12. Prewitt, pseudo-orthophoto case.
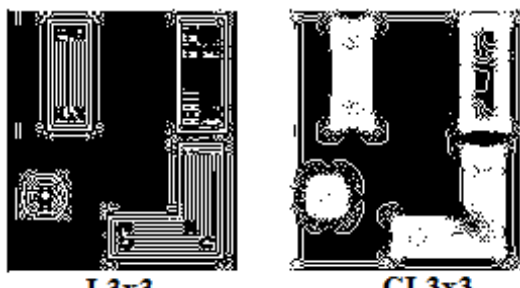

Fig. 13. Laplacian 3x3, pseudo-LiDAR data case

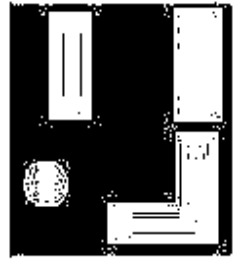

L5x5

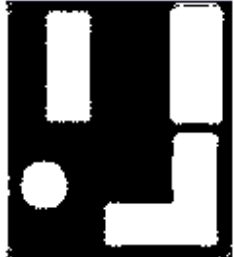

GL5x5
Fig. 14. Laplacian 5x5, pseudo-LiDAR data case.
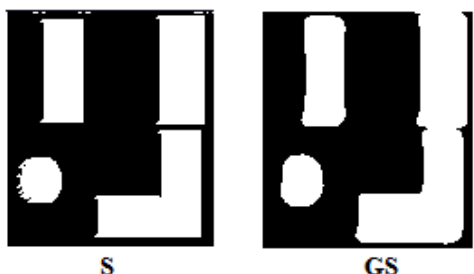

Fig. 15. Sobel, pseudo-LiDAR data case.
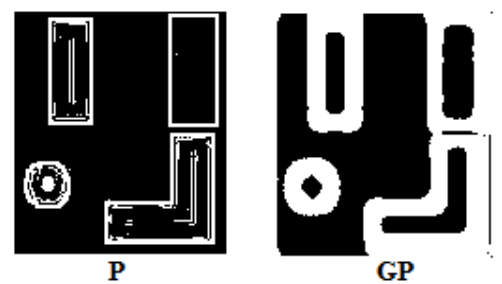

Fig. 16. Prewitt, pseudo-LiDAR data case.

\section{CONCLUSIONS}

The results of the experiment have shown, that transformed LiDAR data is more efficient for the urban objects segmentation, because it does not contain shadows (see in Fig. 9-16). In Fig. 9-12 one can see, that edge detection is sensitive to shadows, it separates light and dark parts of image.

\section{REFERENCES}

[1] I. Dowman, Integration of LiDAR and IfSAR for Mapping: XXth ISPRS Congress Technical Commission II, July 12-23, 2004, Turkey, Istanbul.

[2] W. Li, Q. Guo, M. K. Jakubowski and M. Kelly, "A New Method for Segmenting Individual Trees from the Lidar Point Cloud," Photogrammetric Engineering \& Remote Sensing, vol. 78, pp. 75-84, January 2012. 
[3] N. Senthilkumaran and R. Rajesh, "Edge Detection Techniques for Image Segmentation - A Survey of Soft Computing Approaches," International Journal of Recent Trends in Engineering, vol. 1, pp. 250-254, May 2009.

[4] S. S. Al-amri, N. V. Kalyankar and S. D. Khamitkar, "Image Segmentation by using Edge Detection," International Journal on Computer Science and Engineering, vol. 2, pp. 804-807, 2010.

[5] Y. Ramadevi, T. Sridevi, B. Poornima and B. Kalyani, "Segmentation and Object Recognition using Edge Detection Techniques," International Journal of Computer Science \& Information Technology, vol. 2, pp. 153-160, December 2010.
[6] J.-Y. Rau, N.-Y. Chen and L.-C. Chen, "True Orthophoto Generation of Built-Up Areas Using Multi-View Images," Photogrammetric Engineering \& Remote Sensing, vol. 68, pp. 581-588, June 2002.

[7] R. B. Smith, "Introduction to Remote Sensing of Environment (RSE),"MicroImages, 2012. [Online]. Available: MicroImages, http://www.microimages.com/documentation/ [Accessed March 7, 2013].

[8] S. Martinez and D. Mollicone, "From Land Cover to Land Use: A Methodology to Assess Land Use from Remote Sensing Data," Remote Sensing, vol. 4, no. 4, p. 1024, April 2012. Available: http://www.mdpi.com/journal/remotesensing [Accessed March 7, 2013]. 